\title{
Intelligent Traffic Light Controlling System According to the Traffic Area
}

\section{Randima Fernando ${ }^{1}$ and Anusha Jayasiri ${ }^{2}$}

\begin{abstract}
Traffic congestion is a significant problem in recent years because of the everincreasing number of vehicles in the roads and the poor management of traffic. Traffic congestions are not constant throughout the day. They are changing from time to time. Present traffic controllers have fixed time intervals for red, yellow and green signal lights and therefore, cannot provide a better solution for the dynamic traffic congestion during the day. Computer vision technology can be used to create an intelligent traffic controlling system which can adapt its time intervals according to the real traffic. In the existing traffic controlling systems, a wastage of the green signal duration occurs as fixed green signal duration assigned for a phase is sometimes larger than the actual requirement. Hence, the other roads at the intersection have to wait, in vain, with more traffic, until that fixed green time period is over. In the proposed method, real time traffic image sequences are analyzed by using image processing in order to obtain the actual traffic area. Then, time for green light is assigned according to that traffic area. Hence, the wastage of green signal duration is eliminated by the proposed method since it allocates time for the green signal that is sufficient for the actual traffic
\end{abstract}

1 Department of Statistics and Computer Science, University of Kelaniya,

Sri Lanka, randi.fernando111@gmail.com.

2 Information Technology Centre, University of the Visual and Performing Arts, SriLanka,anusha.j@vpa.ac.lk

Date Received: $\quad 02^{\text {nd }}$ November 2018

Date Accepted: $14^{\text {th }}$ November 2019 
present on the road to the pass. The results reveal that the green signal duration that needs to pass the traffic is proportional to the road area covered by traffic at that time.

Keywords - Traffic area, Image processing, Intelligent controlling of traffic, Green signal duration

\section{Introduction}

Traffic congestion has become a major problem in recent years. It causes many problems such as air pollution, sound pollution, stress and, time and energy wastage. The reasons behind this problem are the insufficiency of the resources provided by the current infrastructure for transportation to meet the ever increasing number of vehicles on the road and the improper controlling of traffic creating traffic jams (Agrawal D. and Sahu A., 2015). These traffic jams not only affect the human routine but also lead to a rise in the cost of transportation.

Traffic congestion in urban areas especially in Colombo is a major problem which needs to be urgently solved. The traffic congestion during a day is dynamic. The current system of fixed time sequenced signal lights cannot adapt its time intervals according to the changing traffic.

There are situations in cross way intersections such as heavy incoming traffic available only from several sides of the intersection while the rest are relatively empty. In this case, people on the heavily - occupied side have to wait for a longer time while the road containing low traffic displays a green signal without having vehicles to move. The allocation of fixed green light time for all sides, leads to a wastage of green signal duration as well as creating more traffic in other roads thereby increasing the average waiting time of every person in the traffic.

The government officials set these timers according to the proportionate amount of traffic present on different sides of the intersection using some statistical data. However, this can never be so flexible to control dynamic traffic throughout the day because some areas of high traffic may receive scanty traffic at some point of the day and some low traffic volume areas might get congested. 
Hence, there is a dire need for a smart system that can adjust the timing of these lights based on the real time traffic present on the road. Because of this, there is an interest in developing intelligent traffic controllers using various technologies such as Magnetic Loop Detectors, Inductive loop detectors, light beams of infrared rays and LASER, and also using image processing.(Choudekar P. et al,2011) Using image processing operations to develop a self-adaptive, intelligent system which can help in better traffic management is cost effective as cameras are cheaper and affordable devices compared to any other devices such as sensors. (Joshi A. A. and Mishra D., 2015)

Several researches have been done for controlling traffic using different techniques of image processing. They are mainly based on vehicle counting methods (Aher C. and Shaikh S.,2015), (Abbas N., et al, 2013) (Niksaz P., 2012) and image comparison methods. (Choudekar P. et al,2011)

The aim of this research is to identify a technologically advanced, intelligent traffic controlling method by recognizing the actual area of the traffic which is presented on a road thereby providing an adequate amount of time for the traffic to pass by dynamically changing traffic light timers.

Moreover, the proposed method is supposed to eliminate the wastage of green signal duration by providing only a sufficient time interval rather than providing a pre-set time interval. Thus, the road with less traffic will be given the green signal for a shorter period of time, while the road with more traffic will be given a longer green signal period.

The ultimate objective of this research is to decrease the average waiting time of people in the traffic thereby saving more energy and time. 


\section{Methodology}

The proposed approach to the elimination of the wastage of 'green signal duration' and controlling traffic is as follows: firstly, a camera was installed at the phases of an intersection to monitor the incoming traffic. After collecting image data sequences through the camera, the area covered by the vehicles in that particular road phase was quantified by processing images. Traffic signal time duration for effective green period will be dynamically set based on the current incoming traffic area from that direction. In this method, the road which

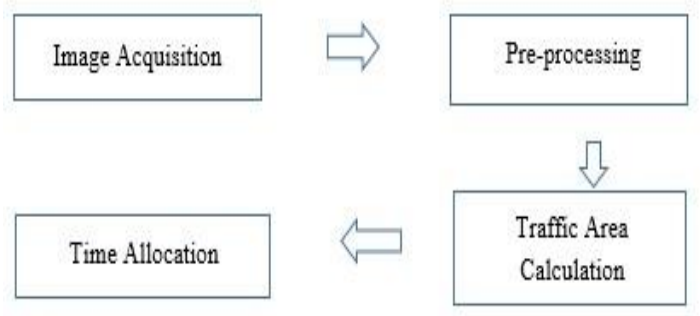

is having more traffic, will be allotted a longer duration of green signal duration compared to others. Hence, it can be used to avoid the wastage of green signal duration.

Figure 1. Proposed method for calculating the green signal duration

According to Figure 1, the proposed method can be divided into four parts: the first part is the acquiring of image sequences of the traffic on the road phase by using a fixed camera. The second part is applying pre-processing techniques on image sequence to enhance the features of the image to prepare it for further analysis. This is achieved by using an OpenCV vision library. The third part is to detect the targeted area where the vehicles are actually presented on the road. The last part is allocating 'effective green signal time' to the phase which can be performed according to the current area of the traffic which covers the road at that particular moment. This research is done with the help of Open Source Computer Vision Library (OpenCV) version 3.2 and $\mathrm{C}++$ with the use of Visual studio 2015. Traffic videos were captured by using a phone camera with 12 MP. 


\section{A. Image Acquisition}

Image acquisition in image processing can be broadly defined as the action of retrieving an image from some source usually a hardware-based source which can be passed through whatever processes that need to occur afterwards. Image acquisition is the first stage of any vision system because without an image, no processing is possible. The image acquired is completely unprocessed. Therefore, after obtaining the image, various methods of image processing are applied to the image to perform many different vision tasks. However, if the image is not acquired satisfactorily, then, the intended tasks may not be achievable even with the aid of image enhancement.

In this research, image sequences were captured by using a phone camera and the camera was stationary. The camera was positioned at sufficient height in order to have a clear view of the road from the point of the traffic lights. The camera is activated a few seconds before the light turns into green. As a preliminary task, offline videos which were taken from real time videos were used to propose a method. The method that is proposed here can be directly applied to the real time videos as well.

The next stage is to extract the frames continuously from the real time video coming from the stationary camera. These frames were then pre-processed and analyzed to detect the traffic area.

\section{B. Pre-processing}

Pre-processing is a common name for operations with images at the lowest level of abstraction. In these operations, both input and output are intensity images. The aim of preprocessing is to suppress unwanted distortions and enhance image features which are important for further processing (Gaikwad O., et al,2014). In other words, pre-processing is done to get a clear image. Since the images are extracted from real time video frames, they can be distorted, blurred, dark etc. For example, images can be blurred in rainy weather. Similarly, images can be darker when captured at night time conditions or can be too bright when it is very sunny. Therefore, different pre-processing methods are applied to the images to improve 
the quality of the image that further helps in better analysis of the image and also the traffic area calculation.

Image pre-processing methods can be categorized according to the size of the pixel neighborhood that is used for the calculation of new pixel brightness. Every piece of video will need some preprocessing to some extent and the amount is wholly dependent on both the source video and the format. The following are some pre-processing techniques used in the proposed method:

1. Image cropping

2. Thresholding

3. Colour space conversion

4. Distance transformation

5. Normalization

1). Image Cropping: Cropping an image extracts a rectangular region of interest from the original image. This allows to zoom in on a specific portion of the image. In order to do cropping, the co-ordinates of the rectangle should be specified. In this research, the coordinate values of the road area are predefined by selecting appropriate values that can extract the whole region of the road. Since the camera is stationary, unnecessary information is fixed in every frame of the live video. Therefore, it is easier to crop the region of interest or the road in every frame.

Image cropping was done using image cropping function in OpenCV.

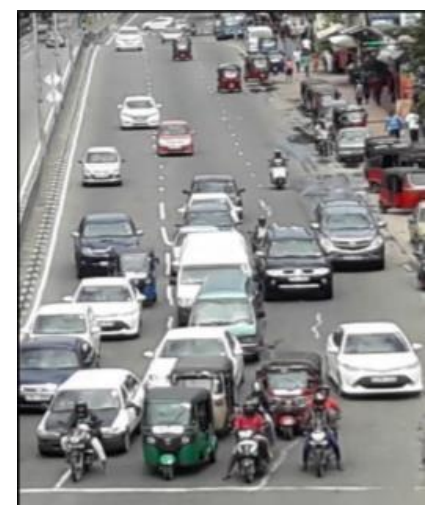


Figure 2. Resultant image after applying image cropping

2). Thresholding: Image thresholding is a simple, yet effective way of partitioning an image into a foreground and a background. This image analysis technique is a type of image segmentation that isolates objects by converting grayscale images into binary images. Thresholding often provides an easy and convenient way to perform this segmentation on the basis of the different intensities or colours in the foreground and background regions of an image. In many vision applications, it is useful to be able to separate the regions of the image corresponding to objects. (Open Source Computer Vision, 2016)

The input to a thresholding operation is typically a grayscale or colour image. In the simplest implementation, the output is a binary image representing the segmentation.

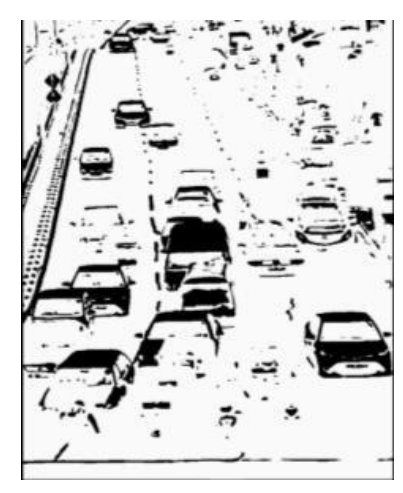

Figure 3. Resultant image after applying adaptive thresholding

3). Colour Space Conversion: The Colour of an image may contain a great deal of information which can be used for simplifying image analysis, object identification and extraction based on colour. These procedures are usually carried out by considering the pixel values in the colour space in which it is defined. To specify a colour in terms of three or more particular characteristics, there are a number of methods called colour spaces or colour models. Selecting a method out of them to represent an image is depended on the requirements of the operations to be performed because some methods are more appropriate according to the 
requirements of applications, for example, in some colour spaces such as RGB, the brightness affects the three channels, a fact that could be unfavorable for some image processing operations.

There are more than 150 colour-space conversion methods available in OpenCV. The function provided by OpenCV in the imgproc module is,

void cvtColor(InputArray src, OutputArray dst, int code, int dstCn=0). (Open Source Computer Vision, 2016)

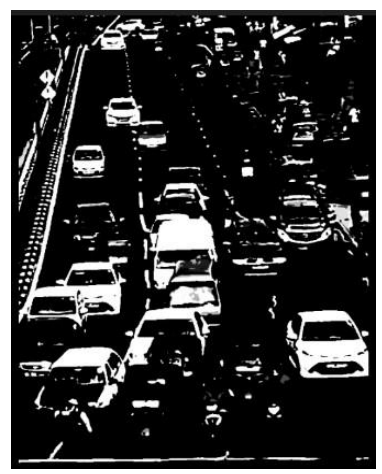

Figure 4. Resultant image after applying color space conversion

4). Distance Transformation: This section describes the distance transformation used for calculating the distance to an object. The distance transform is an operator normally only applied to binary images. The result of the transformation is a grey level image that looks similar to the input image except that the grey level intensities of points inside foreground regions are changed to show the distance to the closest boundary from each point.

There are many ways of computing the distance transformation of a binary image. One intuitive but extremely inefficient way of doing this is to perform multiple successive erosions with a suitable structuring element until all foreground regions of the image have been eroded away. Further, distance transformation can be calculated much more efficiently using clever algorithms which are based on recursive morphology. (Open Source Computer Vision, 2016) 


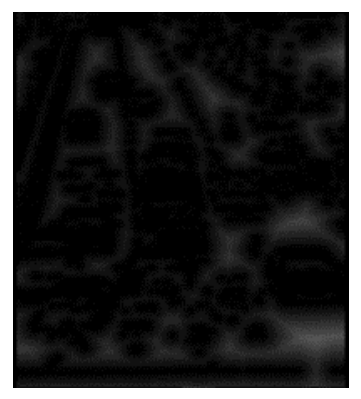

Figure 5. Resultant image after applying distance

5). Normalization: In image processing, normalization is a process that changes the range of pixel intensity values. Normalization is sometimes called as contrast stretching or histogram stretching. In more general fields of data processing such as digital signal processing, it is referred to as dynamic range expansion. The purpose of dynamic range expansion in the various applications is usually to bring the image or other type of signal into a range that is more familiar or normal to the senses. Hence, the term normalization.

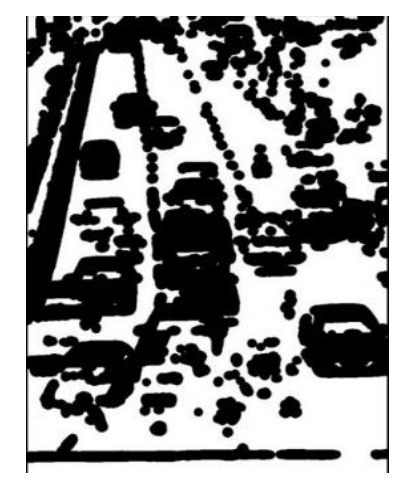

Figure. 6 Resultant image after applying normalization

\section{Traffic Area Identification}

After performing pre-processing techniques described above, the resulting image is ready for further analysis in order to identify the actual area of the road that is covered by vehicles. This study was carried out for a single lane. During the analysis stage, the value of pixels is scanned across straight lines originating from 
the beginning of the starting point of the traffic lights going right until the end of the road. This was achieved with the use of coordinates values of the cropped image.

While scanning each pixel lying on these lines, it is required to identify whether that pixel represents a part of a vehicle or not.

When there is lesser traffic on the road after some point along the road, the values of consecutive pixels hold a value which equals to the pixel value of the road. That is because from that point onwards no vehicle queue is presented on the road or the next vehicle might not still connect the queue as it is still coming or has some distance to pass in order to connect the queue. Hence, the queue length can be derived from the distance between the starting point of the traffic lights and the last co-ordinate of pixel of which the pixel value represents a vehicle.

A similar process was carried out across various lines on the road and then, returned to the length of the line which has the highest distance. This process was applied to all frames in the image sequence to take the highest distance of the line. This distance was used to calculate the traffic area. The traffic area identification at several traffic situations by using the proposed method of this study is illustrated by figure. 7 , figure. 8 and figure. 9 . 


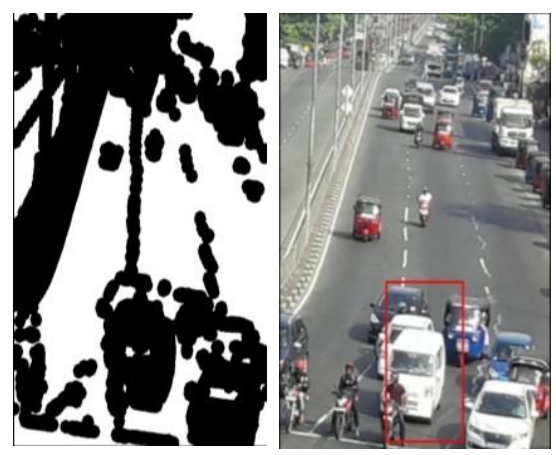

Figure 7. Traffic area identification at low traffic situation

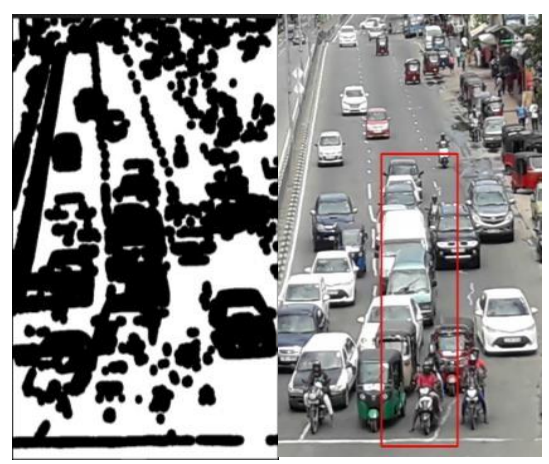

Figure 8. Traffic area identification at medium traffic situation

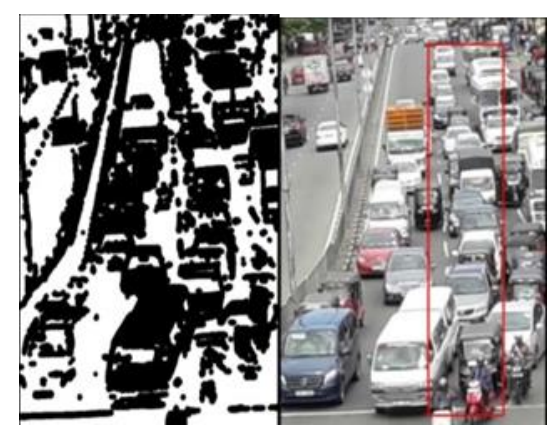

Figure 9. Traffic area identification at high traffic situation 


\section{Determination of green time}

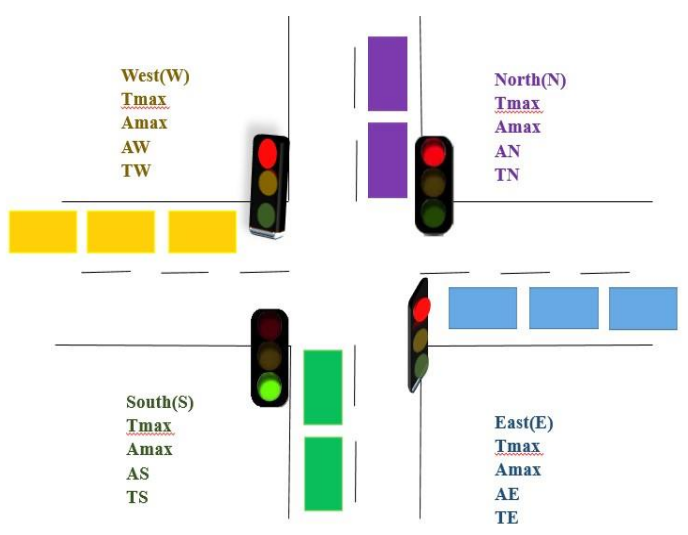

Figure 10. Cross-way intersection

For the time allocation, the work of N. Mokashi. (Mokashi N. 2015) was referred. Consider that the four-way intersection depicted in figure 10 shows the four phases along the directions, north $(\mathrm{N})$, east $(\mathrm{E})$, south (S) and west (W) respectively.

The cycle is defined as the time duration between the beginning of two consecutive green signal durations of the same signal.

Let each road along the directions of $\mathrm{N}, \mathrm{E}, \mathrm{S}$ and $\mathrm{W}$ have equal, maximum green signal duration Tmax and during that time period, the maximum traffic area Amax can pass the intersection point.

Let $A N, A E, A S$ and $A W$ be the areas of traffic which are actually present at that particular moment, measured in the directions of $\mathrm{N}, \mathrm{E}, \mathrm{S}$ and $\mathrm{W}$ respectively.

Let $T N, T E, T S$ and $T W$ be the duration for which the signal turns green for the directions of $\mathrm{N}, \mathrm{E}, \mathrm{S}$ and $\mathrm{W}$ with respect to $A N, A E, A S$ and $A W$ traffic areas at a particular moment. 
Then, we can derive $T N, T E, T S$ and $T W$ as follows:

$$
\begin{aligned}
& T N=\operatorname{Tmax} \times(A N / A \max )-------(2) \\
& T E=T \max \times(A E / A \max )--------(3) \\
& T S=T \max \times(A S / A \max )---------(4) \\
& T W=\operatorname{Tmax} \times(A W / A \max )------(5)
\end{aligned}
$$

According to this method, the green time durations are determined as a portion of Tmax. And also, it can be seen that at each and every cycle, the cycle time is not a constant. It also changes its value according to the area of traffic presented.

Cycle Time $(C)=T N+T E+T S+T W$

$$
=\left(T_{\max } *\left(\frac{A N}{A \max }\right)\right)+\left(T_{\max } *\left(\frac{A E}{A \max }\right)\right)+\left(T_{\max } *\right.
$$

$$
\begin{aligned}
& \left.\left(\frac{A S}{A \max }\right)\right)+\left(T_{\max } *\left(\frac{A W}{A \max }\right)\right) \\
& =T_{\max } *\left(\frac{A N+A E+A S+A W}{A \max }\right)-
\end{aligned}
$$




\section{Results}

Following are the green signal duration based on the several different traffic situations: the time represented here is in seconds.

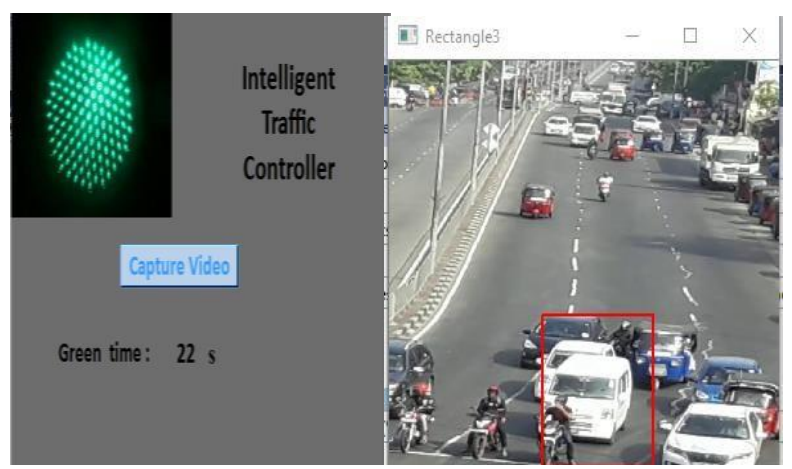

Figure 11. Time allocation at low traffic situation

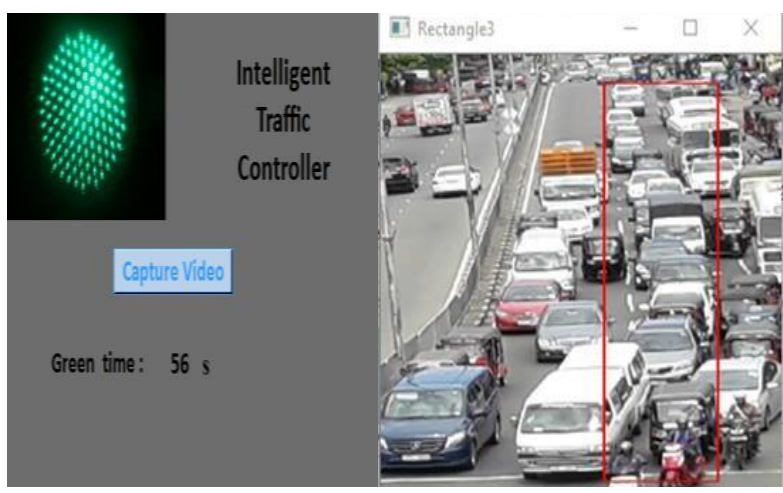

Figure 12. Time allocation at medium traffic situation

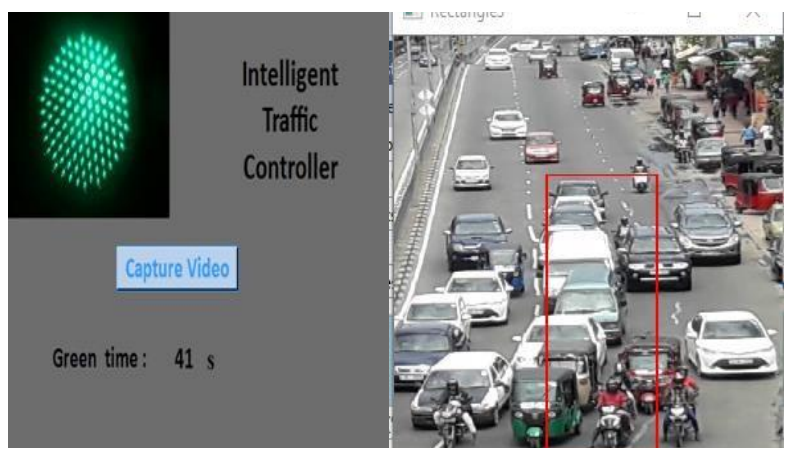

Figure 13. Time allocation at high traffic situation 
duration for this road is 60 seconds. When there was high traffic, these 60 seconds are completely used by vehicles to pass the intersection point. But, in other traffic situations such as low traffic, some portion of this time was sufficient.

In this study, we assumed that this 60 second time is enough to pass the traffic within a maximum area of $200 * 945$ pixels. After obtaining the actual traffic area (that area was also obtained in pixels) by using the method which is described above the green signal duration was determined as a portion of 60 seconds.

The following table shows some traffic area measurements and respective green time durations which were obtained using the proposed method:

\begin{tabular}{|c|c|}
\hline Traffic Area $\left(\right.$ Pixel $\left.^{\mathbf{2}}\right)$ & Effective Green Time (s) \\
\hline 69000 & 22 \\
\hline 70600 & 22 \\
\hline 127800 & 41 \\
\hline 128000 & 41 \\
\hline 139600 & 44 \\
\hline 146200 & 46 \\
\hline 159000 & 50 \\
\hline 159400 & 51 \\
\hline 176400 & 56 \\
\hline
\end{tabular}

Table 1. Traffic area measurements and respective green signal durations 
The following Figure 14 depicts the relationship between the traffic area and the green signal duration which was generated by using this method:

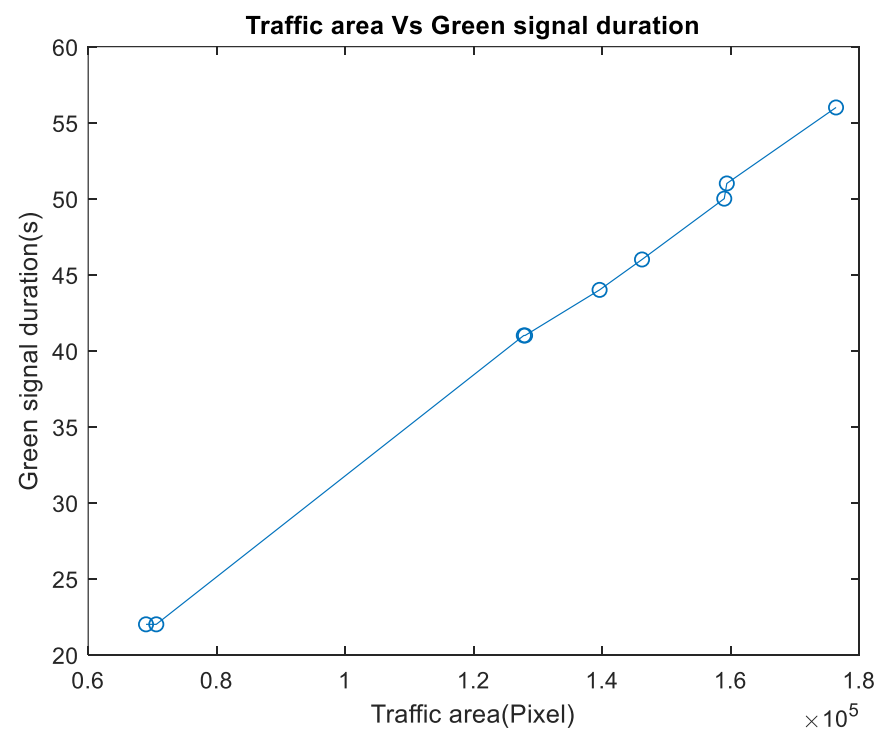

Figure 14. Relationship between traffic area and green signal duration

It is clear that the effective green light time was proportionate to the traffic area. Hence, it can be concluded that the proposed method is accurate. The proposed system optimizes the average waiting time of every person present in the traffic. It can generate the green signal duration intelligently by considering the actual traffic present on the road.

A simple analyzing method was used for analyzing the binary image in order to detect the traffic length. Moreover, a simple calculation was required to obtain the traffic area. Thus, even with less computing power, this method could work well.

The proposed system is very cost-effective compared to the other methods which are used in traffic controlling as it only needs a computer and a digital camera. Furthermore, the cost for Installation and maintenance is less than other technologies.

\section{Discussion}


Traffic congestion is becoming a serious issue day by day since it can lead to many other problems. The main reasons for traffic congestion problems are insufficient infrastructure for transportation with the ever-increasing number of vehicles and inefficient transport management systems etc.

The current traffic controlling systems have been unable to provide efficient solutions for the ever- increasing traffic congestion. The main reason behind the failure of fixed time controllers, is that they could not adapt its time intervals according to the varying traffic patterns. Therefore, the waiting time of each vehicle in the traffic queue is increasing. This study showed that the image processing is a better technique to control the traffic congestion problem. It is also more consistent in detecting vehicle presence because it uses the actual traffic frames. Since it visualizes the reality, it also functions much better than those systems that rely on the detection of vehicles using a metal detector.

The method proposed in this study is control traffic in an intelligent way. It would basically reduce the vehicle waiting time at junctions. It can identify the actual area of traffic with which the road is covered, by using simple methods and it can allocate time for green lights based on the factor of the current traffic area. Thus, a road with less traffic will be given the green signal for a shorter period of time while a road with more traffic will be given the green signal for a longer period. This system is initially developed for controlling traffic in a single lane. It can be further developed for handling traffic in a road with several lanes.

The accuracy of this method can be improved by concurrent analyzing of traffic videos from two points along the road thereby taking the mean value of the area covered by traffic. During the study, it is ascertained that the camera position is very crucial in getting accurate data. A higher camera position is necessary to detect the traffic area accurately.

It is a timely requirement to have the type of system mentioned in this study as this time saving factor will lead to efficient transporting, benefitting not only the vehicle users but also governmental and non-governmental sectors of the country (Rane R., Pathak S., Oak A. and Khachane S.,2015). Furthermore, saving fuel would benefit the entire vehicle users since the money that would be spent on fuel 
will be reduced. Since the vehicles would spend lesser time at the intersections, a more eco-friendly environment will be created thereby lifting the living standards of the people.

\section{Conclusion}

The results show that this method has acquired its objectives by successfully eliminating the wastage of effective green time and controlling traffic in an intelligent way. This method provides an efficient solution regarding the traffic congestion problem. The proposed method is beneficial and with some improvements, it can be used to create a fully completed intelligent traffic controller which can adapt its time intervals according to real time traffic present on the road.

This work can be enhanced further by proposing a system which identifies the presence of emergency vehicles such as ambulances or fire brigades and give preference to those lanes with emergency vehicles to pass the traffic (Khanke P. and Kulkarni S., 2014).

In addition, any future work can involve further image processing that would provide better results including night time images. Through these means, traffic can be controlled in the day time as well as night time.

In addition, this work can be further expanded by implementing this technique with the real time online image sequences and can be used to create a perfect traffic controlling system.

\section{References}


Abbas N., Tayyab M., and Qadr M., 2013. Real Time Traffic Density Count using Image Processing, International Journal of Computer Applications, 83.

Agrawal D. and Sahu A., 2015. Intelligent Real Time Traffic Controller Using Image Processing, International Journal of Science and Research ,4(4).

Aher C. and Shaikh S.,2015. Adaptive Traffic Control and Traffic Density Monitoring System using an Image Processing, International Journal of Advanced Research in Electrical, Electronics and Instrumentation Engineering, 4(11).

Joshi A. A. and Mishra D., 2015. Review of Traffic Density Analysis Techniques, International Journal of Advanced Research in Computer and Communication Engineering, 4(7).

Choudekar P., Banerjee S. and Muju M.,2011. Real Time Traffic Light Control Using Image Processing, Indian Journal of Computer Science and Engineering,

Dangi V., Parab A., Pawar K. and Rathod S., 2012. Image Processing Based Intelligent Traffic Controller, Undergraduate Academic Research Journal,1(1).

Gaikwad O., Vishwasrao A., Pujari K. and Talathi T.,2014. Image Processing Based Traffic Light Control. International Journal of Science, Engineering and Technology Research, 3(4).

Khanke P. and Kulkarni S., 2014. A Technique on Road Traffic Analysis using Image

Processing. International Journal of Engineering Research \& Technology,3(2).

Mokashi N., 2015. Intelligent Traffic Signal Control using Image Processing. International Journal of Advance Research in Computer Science and Management Studies, 3(10).

Niksaz P., 2012. Automatic Traffic Estimation Using Image Processing. International Conference on Image, Vision and Computing (ICIVC 2012), 50. 
Rane R., Pathak S., Oak A. and Khachane S.,2015. Real Time Traffic Control using Image Processing. International Journal for Scientific Research \& Development,3(3).

Open Source Computer Vision (2016), viewed 22 September 2018, https://docs.opencv.org/3.2.0/ 